\title{
The Effects of Geriatric Depression on Suicide Intention: The Moderating Effects of Positive Psychological Capital
}

\author{
Soon-man Kwon ${ }^{1}$, Jea-ug Ko ${ }^{2}$ \\ ${ }^{1}$ Part-time Instructor, Dept. of Social Welfare, Catholic Kwandong University, Korea, \\ sm23@hanmail.net \\ ${ }^{2}$ Professor, Dept. of Social Welfare, Catholic Kwandong University, Korea, kojea62@cku.ac.kr
}

Corresponding author: Jea-ug Ko

\begin{abstract}
The purpose of this study was to provide primary data that could improve the quality of life and prevent suicide incidence in the elderly group. A survey was conducted on 746 persons aged 65 and above at senior welfare centers and local community centers for the elderly in S City and G and G Provinces. The subjects were measured for geriatric depression, suicide intention, and positive psychological capital and the following results were obtained: First, the more depressed the subject was, the stronger the subject's intention to commit suicide was. Second, positive psychological capital partially affected the suicide intention. Third, depression and positive psychological capital produced partial effects. Lastly, the association between geriatric depression and suicide intention, along with the moderating effects of positive psychological capital, which tends to serve as a buffer between the two variables, were demonstrated. This study is significant because it determined the association between the effects of geriatric depression on suicide intention and positive psychological capital and demonstrated the importance of positive psychological capital in preventing suicide in the elderly population. The findings are expected to provide basic data that can help develop interventional schemes and policies for elderly suicide prevention by presenting the moderating effects of positive psychological capital.
\end{abstract}

Keywords: Geriatric Depression, Suicide Intention, Positive Psychological Capital, Depression

\section{Introduction}

\subsection{Need for Study}

As South Korea is entering an aging society in terms of the population structure, it is faced with a great deal of social problems at the macroscopic level, such as the aging labor population, the decrease in the effectual demand, and the increase in support for seniors, which lead to intergenerational conflicts. In particular, the issue of mental health, being related to depression and among the social problems of seniors, is a serious subject[1].

Due to these concerns, South Korea has an elderly suicide rate of $28.7 \%$, dishonorably standing first among the OECD countries[2]. In 2020, Statistics Korea indicated that health and financial problems were two of the most significant issues people aged 65 and above experienced[3]. There were much more complex and varied causes of suicide in the elderly group than in the other age groups. The most frequent reason for elderly suicide intention was financial hardship $(27.7 \%)$, followed by their health problems $(27.6 \%)$, conflicts or isolation from spouse or children $(18.6 \%)$, loneliness $(12.4 \%)$, death of

*This is an adaptation of the first author's doctoral thesis.

Received: October 15, 2020; 1st Review Result: December 02, 2020; 2nd Review Result: January 15, 2021

Accepted: February 26, 2021 
an intimate person $(8.3 \%)$, health problems of spouse or family $(4.9 \%)$, and others $(0.5 \%)[4]$. A literature review has found that depression is an essential predictor of elderly suicide intention[5-7]. Taking this into account, it can be said that geriatric depression is closely associated with suicide intention.

Positive psychotherapy has been improved for about 20 years since Seligman founded positive psychology in 1998, and it has recently drawn much attention as it was also found to be effective in relieving and preventing depression[8]. Positive psychology attaches importance to individuals' growth initiative and hope, and believes that they can enjoy a joyful life through self-improvement to clarify their values and make their life more meaningful[9]. It can be said that both - whether they can continuously improve their daily lives even under the conditions of stress and physical and mental distress in old age and whether they can overcome depression through positive perception, attitude formation, and effective management of emotions - are crucial.

As depression was a significant risk factor for suicide intention, research has been conducted on the variables relieving the adverse effects of depression, leading to suicide intention. However, no research has been conducted on perceived positive psychological capital as a variable moderating the association between geriatric depression and suicide intention. As positive psychological capital can maximize personal potential through the positive elements and strengths of ordinary people rather than through the modification of the negative aspects, the fact that it draws attention as an alternative to overcome the limitations of the existing human resource development seems to be meaningful in preventing geriatric depression and suicide intention[10].

As geriatric depression has risen as a crucial element that lowers the quality of life for seniors in the aging society, it is becoming one of the most critical social problems, in addition to poverty, disease, solitude, and suicide in this society. Thus, This study was conducted based on the belief that analyzing the association between geriatric depression and suicide intention is an essential task to improve seniors' quality of life. This study aims to analyze the effects of geriatric depression on suicide intention, take the moderating effects of positive psychological capital into account, and provide basic data to improve seniors' quality of life.

\subsection{Questions}

This study aims to analyze the moderating effects of positive psychological capital on geriatric depression and, thereby, on suicide intention. For this purpose, the following questions were set:

First, how are the effects of geriatric depression on suicide intention correlated with positive psychological capital?

Second, does positive psychological capital have any moderating influence on how geriatric depression affects suicide intention?

\section{Methods}

\subsection{Subjects}

A survey was conducted on 800 persons aged 65 and above residing in S City and G and G Provinces from March 2 to 24, 2017. For data collection, a letter for cooperation was sent to senior welfare centers in S City and G Province, and a full explanation regarding the research was given to the social workers at the centers that gave their consent before the face-to-face survey. The presidents of local community centers for the elderly were asked to cooperate with the researcher, and a personal visit was made to those who gave their consent for the face-to-face survey. A personal visit was also made to elderly shelters for the face-to-face survey. 
Every respondent was given a full explanation of the research's contents and purpose and notified that personal information related to the responses would be thoroughly kept confidential before the survey. Of 800 questionnaires, 746 were finally analyzed, counting out 54 containing substantial missing data.

\subsection{Instruments}

The Korean version of CES-D standardized by Jeon[11] was used to measure geriatric depression. While this scale was composed of 20 items, 18 items were used, except 2, for this study. It was composed of three factors (personal, environmental, and others) so that they could give an answer on the 5-point Likert scale regarding depressive symptoms they had experienced for the past week. It contained four positive items; the higher the score in back-calculation, the more depressed. The instrument translated and applied by Shin[12] was used to measure elderly suicide intention. This selfreporting questionnaire was transformed to contain 19 items on the 3-point (0-2) Likert scale, with a total score ranging from 0 to 38 . The higher the sum of scores for 18 items, the higher the suicide intention. As for the total score, $\leq 8$ was categorized into no risk of suicide, 9-11 into the low risk of suicide, 12-14 into the intermediate risk of suicide, and $\geq 15$ into high risk of suicide. Luthans's[13] inventory with a total of 24 items - 6 for each construct - was used to measure positive psychological capital for seniors. In this study, a total of 20 items -5 for each construct - were used to meet the purpose.

\subsection{Reliability}

As for the reliability of the variables, depression was very reliable (0.816), as were its sub-variables $(0.680-0.889)$. Suicide intention was very reliable (0.873). Positive psychological capital was very reliable (0.923), and its sub-variables were very reliable (0.805-0.822). The results of the reliability analysis are presented in [Table 1].

[Table 1] Reliability Test of Variables

\begin{tabular}{|c|c|c|c|}
\hline \multicolumn{2}{|c|}{ Division } & \multicolumn{2}{|c|}{ Cronbach's Alpha } \\
\hline \multirow{3}{*}{ Depression } & Self-depression & .889 & \multirow{3}{*}{.816} \\
\hline & Environmental depression & .689 & \\
\hline & Others' depression & .680 & \\
\hline \multirow{4}{*}{$\begin{array}{c}\text { Positive psychological } \\
\text { capital }\end{array}$} & Self-efficacy & .822 & \multirow{4}{*}{.923} \\
\hline & Hope & .805 & \\
\hline & Optimism & .807 & \\
\hline & Resilience & .809 & \\
\hline \multicolumn{2}{|c|}{ Suicide intention } & \multicolumn{2}{|c|}{.873} \\
\hline
\end{tabular}

\subsection{Data Processing and Analysis}

The data were coded and statistically processed using SPSS ver 19.0, with the significance level set at $\mathrm{p}<0.05$. Stepwise regression analysis was performed for the moderating effects in addition to multiple regression analysis for each instrument. 


\section{Results}

\subsection{Correlation Between the Effects of Geriatric Depression on Suicide Intention and Positive Psychological Capital Data Processing and Analysis}

Correlation analysis was performed using Pearson's correlation coefficient for inter-variable correlation. Depression and suicide were negatively correlated with positive psychological capital, while depression was positively correlated with suicide; that is, there was a correlation among most variables. This is consistent with the direction of the model in this study, and the results are presented in [Table 2].

[Table 2] Correlation Analysis of Variables

\begin{tabular}{|c|c|c|c|c|c|c|c|c|c|}
\hline \multicolumn{2}{|c|}{ Division } & $\begin{array}{c}\text { Self- } \\
\text { depressi }\end{array}$ & $\begin{array}{c}\text { Environmen } \\
\text { tal }\end{array}$ & $\begin{array}{l}\text { Others' } \\
\text { depressi }\end{array}$ & $\begin{array}{c}\text { Self- } \\
\text { efficac }\end{array}$ & Hope & Optimis & Resilien & $\begin{array}{l}\text { Suicide } \\
\text { intentio }\end{array}$ \\
\hline \multirow{3}{*}{ Depression } & $\begin{array}{c}\text { Self- } \\
\text { depression }\end{array}$ & 1 & & & & & & & \\
\hline & $\begin{array}{c}\text { Environmen } \\
\text { tal } \\
\text { depression }\end{array}$ & $.263 * * *$ & 1 & & & & & & \\
\hline & $\begin{array}{c}\text { Others' } \\
\text { depression }\end{array}$ & $.534 * * *$ & $.185^{* * *}$ & 1 & & & & & \\
\hline \multirow{4}{*}{$\begin{array}{c}\text { Positive } \\
\text { psychologi } \\
\text { cal capital }\end{array}$} & $\begin{array}{c}\text { Self- } \\
\text { efficacy }\end{array}$ & $-157 * * *$ & $-342^{2 * 2 k}$ & .045 & 1 & & & & $-205 * * *$ \\
\hline & Hope & $-173^{* * * *}$ & $-376^{* * 1 * k}$ & -.019 & $\begin{array}{c}.680 * * \\
*\end{array}$ & 1 & & & $-256 * * *$ \\
\hline & Optimism & $-248^{* 216 *}$ & $-270^{2006 k}$ & $-.107 * *$ & $\begin{array}{c}.531^{* *} \\
*\end{array}$ & $\begin{array}{c}.593 * * \\
*\end{array}$ & 1 & & $-218^{* * * * *}$ \\
\hline & Resilience & $-141 * * *$ & $-354 * *$ & .041 & $\begin{array}{c}.659^{* *} \\
*\end{array}$ & $\begin{array}{c}.708^{* *} \\
*\end{array}$ & $.556 * * *$ & 1 & $-179 * * *$ \\
\hline \multicolumn{2}{|c|}{ Suicide intention } & $.215 * * *$ & $.143 * * *$ & $.321 * * *$ & $-205^{* * 1 * k}$ & $-256^{* 2 * k}$ & $-218^{* * * k}$ & $-179 \%$ & 1 \\
\hline
\end{tabular}

${ }^{*} \mathrm{p}<.05,{ }^{*} \mathrm{p}<.01, * * * \mathrm{p}<.001$

\subsection{Association Among Depression, Suicide Intention, and Positive Psychological Capital}

Stepwise regression analysis was performed for the moderating effects of positive psychological capital on depression and suicide intention. The moderating effects involved three stages: at Stage 1, the effects on dependent variables were analyzed by entering independent variables alone; at Step 2, the effects on dependent variables were analyzed by adding moderating variables; at Step 3, it was determined that there were moderating effects when adding an interaction term of independent and dependent variables.

\subsubsection{Analysis of the Moderating Influence of Positive Psychological Capital on Self-Depression's Effects on Suicide Intention}

As for the moderating effects of self-efficacy on self-depression's effects on suicide intention, selfdepression positively affected suicide intention at Stage 1. The regression model's explanatory power increased by $5.9 \%$, with suicide intention positively affected by self-depression and negatively affected by self-efficacy at Stage 2 . The regression model's explanatory power increased by $0.7 \%$, with the interaction between self-depression and self-efficacy negatively affecting suicide intention at Stage 3. Self-efficacy moderated self-depression's effects on suicide intention, as presented in [Table 3]. 
[Table 3] Analysis of the Moderating Influence of Self-Efficacy on Self-Depression's Effects on Suicide Intention

\begin{tabular}{|c|c|c|c|c|c|c|c|}
\hline \multirow{2}{*}{$\begin{array}{c}\text { Dependent } \\
\text { variable }\end{array}$} & \multirow{2}{*}{$\begin{array}{c}\text { Independent } \\
\text { variable }\end{array}$} & \multicolumn{2}{|c|}{ Stage 1} & \multicolumn{2}{|c|}{ Stage 2} & \multicolumn{2}{|c|}{ Stage 3} \\
\hline & & $\boldsymbol{\beta}$ & $\mathbf{t}$ & $\boldsymbol{\beta}$ & $\mathbf{t}$ & $\mathbf{p}$ & $\mathbf{t}$ \\
\hline \multirow{4}{*}{$\begin{array}{l}\text { Suicide } \\
\text { intention }\end{array}$} & (Constant) & & 1.150 & & 5.497 & & .206 \\
\hline & $\begin{array}{c}\text { Self-depression } \\
\text { (A) }\end{array}$ & .215 & $6.001 * * *$ & .255 & $7.244 * * *$ & .536 & $4.191 * * *$ \\
\hline & $\begin{array}{c}\text { Self-efficacy } \\
\text { (B) }\end{array}$ & & & -.246 & $-6.997 * * *$ & -.026 & -.258 \\
\hline & $A * B$ & & & & & -.392 & $-2.287 *$ \\
\hline \multicolumn{2}{|c|}{ R Square } & \multicolumn{2}{|c|}{.046} & \multicolumn{2}{|c|}{.105} & \multicolumn{2}{|c|}{.112} \\
\hline \multicolumn{2}{|c|}{$\triangle R$ Square } & & & \multicolumn{2}{|c|}{.059} & \multicolumn{2}{|c|}{.007} \\
\hline \multicolumn{2}{|c|}{$\mathrm{F}$} & \multicolumn{2}{|c|}{$36.010 * * *$} & \multicolumn{2}{|c|}{$43.644 * * *$} & \multicolumn{2}{|c|}{$31.005 * * *$} \\
\hline
\end{tabular}

$* \mathrm{p}<.05, * * * \mathrm{p}<.001$

As for the moderating influence of hope on self-depression's effects on suicide intention, selfdepression positively affected suicide intention at Stage 1 . The regression model's explanatory power increased by $8.9 \%$, with suicide intention positively affected by self-depression and negatively affected by hope at Stage 2. The regression model's explanatory power increased by $1.1 \%$, with the interaction between self-depression and hope negatively affecting suicide intention at Stage 3. Hope moderated self-depression's effects on suicide intention, as presented in [Table 4].

[Table 4] Analysis of the Moderating Influence of Hope on Self-Depression's Effects on Suicide Intention

\begin{tabular}{|c|c|c|c|c|c|c|c|}
\hline \multirow{2}{*}{$\begin{array}{l}\text { Dependent } \\
\text { variable }\end{array}$} & \multirow{2}{*}{$\begin{array}{c}\text { Independent } \\
\text { variable }\end{array}$} & \multicolumn{2}{|c|}{ Stage 1} & \multicolumn{2}{|c|}{ Stage 2} & \multicolumn{2}{|c|}{ Stage 3} \\
\hline & & $\beta$ & $\mathbf{t}$ & $\beta$ & $\mathbf{t}$ & p & $\mathbf{t}$ \\
\hline \multirow{4}{*}{$\begin{array}{l}\text { Suicide } \\
\text { intention }\end{array}$} & (Constant) & & 1.150 & & 6.528 & & -.084 \\
\hline & $\begin{array}{c}\text { Self- } \\
\text { depression } \\
\text { (A) }\end{array}$ & .215 & $6.001 * * *$ & .269 & $7.750 * * *$ & .637 & $5.083 * * *$ \\
\hline & $\begin{array}{l}\text { Hope } \\
\text { (B) }\end{array}$ & & & -.303 & $-8.749 * * *$ & -.011 & -.106 \\
\hline & $\mathrm{A} * \mathrm{~B}$ & & & & & -.520 & $-3.056 * *$ \\
\hline \multicolumn{2}{|c|}{ R Square } & \multicolumn{2}{|c|}{.046} & \multicolumn{2}{|c|}{.135} & \multicolumn{2}{|c|}{.146} \\
\hline \multicolumn{2}{|c|}{$\triangle \mathrm{R}$ Square } & & & \multicolumn{2}{|c|}{.089} & \multicolumn{2}{|c|}{.011} \\
\hline \multicolumn{2}{|c|}{$\mathrm{F}$} & \multicolumn{2}{|c|}{$36.010 * * *$} & \multicolumn{2}{|c|}{$58.104 * * *$} & \multicolumn{2}{|c|}{$42.286^{* * * *}$} \\
\hline
\end{tabular}

$* * \mathrm{p}<.01, * * * \mathrm{p}<.001$

As for the moderating influence of optimism on self-depression's effects on suicide intention, selfdepression positively affected suicide intention at Stage 1 . The regression model's explanatory power increased by $7.9 \%$, with suicide intention positively affected by self-depression and negatively affected by optimism at Stage 2. The regression model's explanatory power increased by $1.3 \%$, with the interaction between self-depression and optimism negatively affecting suicide intention at Stage 3; that is, optimism moderated self-depression's effects on suicide intention, as presented in [Table 5]. 
[Table 5] Analysis of the Moderating Influence of Optimism on Self-Depression's Effects on Suicide Intention

\begin{tabular}{|c|c|c|c|c|c|c|c|}
\hline \multirow{2}{*}{$\begin{array}{c}\text { Dependent } \\
\text { variable }\end{array}$} & \multirow{2}{*}{$\begin{array}{c}\text { Independent } \\
\text { variable }\end{array}$} & \multicolumn{2}{|c|}{ Stage 1} & \multicolumn{2}{|c|}{ Stage 2} & \multicolumn{2}{|c|}{ Stage 3} \\
\hline & & $\beta$ & $\mathbf{T}$ & $\beta$ & $\mathbf{T}$ & $\mathbf{p}$ & $\mathbf{t}$ \\
\hline \multirow{4}{*}{$\begin{array}{l}\text { Suicide } \\
\text { intention }\end{array}$} & (Constant) & & 1.150 & & 5.743 & & -.969 \\
\hline & $\begin{array}{c}\text { Self- } \\
\text { depression } \\
\text { (A) }\end{array}$ & .215 & $6.001 * * *$ & .288 & $8.110 * * *$ & .716 & $5.412 * * *$ \\
\hline & $\begin{array}{l}\text { Optimism } \\
\text { (B) }\end{array}$ & & & -.290 & $-8.167 * * *$ & .085 & .725 \\
\hline & $\mathrm{A} * \mathrm{~B}$ & & & & & -.646 & $-3.357 * *$ \\
\hline \multicolumn{2}{|c|}{ R Square } & \multicolumn{2}{|c|}{.046} & \multicolumn{2}{|c|}{.125} & \multicolumn{2}{|c|}{.138} \\
\hline \multicolumn{2}{|c|}{$\triangle R$ Square } & & & \multicolumn{2}{|c|}{.079} & \multicolumn{2}{|c|}{.013} \\
\hline \multicolumn{2}{|c|}{$F$} & \multicolumn{2}{|c|}{$36.010 * * *$} & \multicolumn{2}{|c|}{$52.951 * * *$} & \multicolumn{2}{|c|}{$39.547 * * *$} \\
\hline
\end{tabular}

$* * \mathrm{p}<.01, * * * \mathrm{p}<.001$

As for the moderating influence of resilience on self-depression's effects on suicide intention, selfdepression positively affected suicide intention at Stage 1 . The regression model's explanatory power increased by $5.0 \%$, with suicide intention positively affected by self-depression and negatively affected by resilience at Stage 2. The regression model's explanatory power increased by $0.8 \%$, with the interaction between self-depression and resilience negatively affecting suicide intention at Stage 3; that is, resilience moderated self-depression's effects on suicide intention, as presented in [Table 6].

[Table 6] Analysis of the Moderating Influence of Resilience on Self-Depression's Effects on Suicide Intention

\begin{tabular}{|c|c|c|c|c|c|c|c|}
\hline \multirow{2}{*}{$\begin{array}{c}\text { Dependent } \\
\text { variable }\end{array}$} & \multirow{2}{*}{$\begin{array}{c}\text { Independent } \\
\text { variable }\end{array}$} & \multicolumn{2}{|c|}{ Stage 1} & \multicolumn{2}{|c|}{ Stage 2} & \multicolumn{2}{|c|}{ Stage 3} \\
\hline & & $\beta$ & $\mathbf{t}$ & $\beta$ & $\mathbf{T}$ & $\mathbf{p}$ & $\mathbf{t}$ \\
\hline \multirow{4}{*}{ Suicide intention } & (Constant) & & 1.150 & & 4.972 & & -.001 \\
\hline & $\begin{array}{c}\text { Self-depression } \\
\text { (A) }\end{array}$ & .215 & $6.001 * * *$ & .247 & $6.979 * * *$ & .543 & $4.136^{* * * *}$ \\
\hline & $\begin{array}{c}\text { Resilience } \\
\text { (B) }\end{array}$ & & & -.216 & $-6.097 * * *$ & .009 & .089 \\
\hline & $\mathrm{A} * \mathrm{~B}$ & & & & & -.405 & $-2.340 *$ \\
\hline \multicolumn{2}{|c|}{ R Square } & \multicolumn{2}{|c|}{.046} & \multicolumn{2}{|c|}{.092} & \multicolumn{2}{|c|}{.098} \\
\hline \multicolumn{2}{|c|}{$\triangle \mathrm{R}$ Square } & & & \multicolumn{2}{|c|}{.050} & \multicolumn{2}{|c|}{.008} \\
\hline \multicolumn{2}{|c|}{$\mathrm{F}$} & \multicolumn{2}{|c|}{$36.010 * * *$} & \multicolumn{2}{|c|}{37.466 *** } & \multicolumn{2}{|c|}{$26.954 * * *$} \\
\hline
\end{tabular}

${ }^{*} \mathrm{p}<.05, * * * \mathrm{p}<.001$

\subsubsection{Analysis of the Moderating Influence of Positive Psychological Capital on Environmental Depression's Effects on Suicide Intention}

As for the moderating influence of self-efficacy on environmental depression's effects on suicide intention, environmental depression positively affected suicide intention at Stage 1 . The explanatory power of the regression model increased by $2.7 \%$, with suicide intention positively affected by environmental depression and negatively affected by self-efficacy at Stage 2 . The regression model's explanatory power increased by $1.9 \%$, with the interaction between environmental depression and self- 
efficacy positively affecting suicide intention at Stage 3; that is, self-efficacy moderated environmental depression's effects on suicide intention, as presented in [Table 7].

[Table 7] Analysis of the Moderating Influence of Self-Efficacy on Environmental Depression's Effects on Suicide Intention

\begin{tabular}{|c|c|c|c|c|c|c|c|}
\hline \multirow{2}{*}{$\begin{array}{c}\text { Dependent } \\
\text { variable }\end{array}$} & \multirow{2}{*}{$\begin{array}{c}\text { Independent } \\
\text { variable }\end{array}$} & \multicolumn{2}{|c|}{ Stage 1} & \multicolumn{2}{|c|}{ Stage 2} & \multicolumn{2}{|c|}{ Stage 3} \\
\hline & & $\boldsymbol{\beta}$ & $\mathbf{t}$ & $\boldsymbol{\beta}$ & $\mathbf{t}$ & $\mathbf{p}$ & $\mathbf{t}$ \\
\hline \multirow{4}{*}{ Suicide intention } & (Constant) & & 2.799 & & 5.422 & & 5.983 \\
\hline & $\begin{array}{l}\text { Environmental } \\
\text { depression } \\
\text { (A) }\end{array}$ & .143 & $3.949 * * *$ & .083 & $2.191 *$ & -.312 & $-2.834 * *$ \\
\hline & Self-efficacy (B) & & & -.177 & $-4.637 * * *$ & -.610 & $-5.106 * * * *$ \\
\hline & A*B & & & & & .496 & $3.824 * * *$ \\
\hline \multicolumn{2}{|c|}{ RSquare } & \multicolumn{2}{|c|}{.021} & \multicolumn{2}{|c|}{.048} & \multicolumn{2}{|c|}{.067} \\
\hline \multicolumn{2}{|c|}{$\triangle \mathrm{R}$ Square } & & & \multicolumn{2}{|c|}{.027} & \multicolumn{2}{|c|}{.019} \\
\hline \multicolumn{2}{|c|}{$\mathrm{F}$} & \multicolumn{2}{|c|}{$15.596 * * *$} & \multicolumn{2}{|c|}{$18.762 * * *$} & \multicolumn{2}{|c|}{$17.613 * * *$} \\
\hline
\end{tabular}

${ }^{*} \mathrm{p}<.05,{ }^{* *} \mathrm{p}<.01, * * * \mathrm{p}<.001$

As for the moderating effects of hope on the effects of environmental depression on suicide intention, environmental depression positively affected suicide intention at Stage 1; the explanatory power of the regression model increased by $4.7 \%$, with suicide intention negatively affected by hope, at Stage 2. The explanatory power of the regression model increased by $2.5 \%$, with the interaction between environmental depression and hope positively affecting suicide intention, at Stage 3. That is, hope had moderating effects on the effects of environmental depression on suicide intention, as presented in [Table 8].

[Table 8] Analysis of the Moderating Influence of Hope on Environmental Depression's Effects on Suicide Intention

\begin{tabular}{|c|c|c|c|c|c|c|c|}
\hline \multirow{2}{*}{$\begin{array}{l}\text { Dependent } \\
\text { variable }\end{array}$} & \multirow{2}{*}{$\begin{array}{l}\text { Independent } \\
\text { variable }\end{array}$} & \multicolumn{2}{|c|}{ Stage 1} & \multicolumn{2}{|c|}{ Stage 2} & \multicolumn{2}{|c|}{ Stage 3} \\
\hline & & $\beta$ & $\mathbf{t}$ & $\beta$ & $\mathbf{t}$ & p & $\mathbf{t}$ \\
\hline \multirow{4}{*}{$\begin{array}{l}\text { Suicide } \\
\text { intention }\end{array}$} & (Constant) & & 2.799 & & 6.681 & & 7.196 \\
\hline & $\begin{array}{l}\text { Environmental } \\
\text { depression } \\
\text { (A) }\end{array}$ & .143 & $3.949 * * *$ & .055 & 1.447 & -.393 & $-3.672 * * *$ \\
\hline & $\begin{array}{l}\text { Hope } \\
\text { (B) }\end{array}$ & & & -.235 & $-6.149 * * *$ & -.746 & $-6.207 * * *$ \\
\hline & $A * B$ & & & & & .561 & $4.477 * * *$ \\
\hline \multicolumn{2}{|c|}{ R Square } & \multicolumn{2}{|c|}{.021} & \multicolumn{2}{|c|}{.068} & \multicolumn{2}{|c|}{.093} \\
\hline \multicolumn{2}{|c|}{$\triangle R$ Square } & & & \multicolumn{2}{|c|}{.047} & \multicolumn{2}{|c|}{.025} \\
\hline \multicolumn{2}{|c|}{$\mathrm{F}$} & \multicolumn{2}{|c|}{$15.596^{* * *}$} & \multicolumn{2}{|c|}{$27.092 * * *$} & \multicolumn{2}{|c|}{$25.208 * * *$} \\
\hline
\end{tabular}

$* * * \mathrm{p}<.001$

As for the moderating influence of optimism on environmental depression's effects on suicide intention, environmental depression positively affected suicide intention at Stage 1 . The explanatory power of the regression model increased by $3.4 \%$, with suicide intention positively affected by environmental depression and negatively affected by optimism at Stage 2. The regression model's 
explanatory power increased by $2.1 \%$, with the interaction between environmental depression and optimism positively affecting suicide intention at Stage 3; that is, optimism moderated environmental depression's effects on suicide intention, as presented in [Table 9].

[Table 9] Analysis of the Moderating Effects of Optimism on Environmental Depression's Effects on Suicide Intention

\begin{tabular}{|c|c|c|c|c|c|c|c|}
\hline \multirow{2}{*}{$\begin{array}{c}\text { Dependent } \\
\text { variable }\end{array}$} & \multirow{2}{*}{$\begin{array}{l}\text { Independent } \\
\text { variable }\end{array}$} & \multicolumn{2}{|c|}{ Stage 1} & \multicolumn{2}{|c|}{ Stage 2} & \multicolumn{2}{|c|}{ Stage 3} \\
\hline & & $\beta$ & $\mathbf{t}$ & $\beta$ & $\mathbf{t}$ & p & $\mathbf{t}$ \\
\hline \multirow{4}{*}{$\begin{array}{l}\text { Suicide } \\
\text { intention }\end{array}$} & (Constant) & & 2.799 & & 4.748 & & 5.636 \\
\hline & $\begin{array}{c}\text { Environmental } \\
\text { depression } \\
\text { (A) }\end{array}$ & .143 & $3.949 * * *$ & .092 & $2.383^{*}$ & -.305 & $-2.746 * *$ \\
\hline & $\begin{array}{l}\text { Resilience } \\
\text { (B) }\end{array}$ & & & -.147 & $-3.820 * * *$ & -.589 & $-4.814 * * *$ \\
\hline & $\mathrm{A} * \mathrm{~B}$ & & & & & .497 & $3.803 * * *$ \\
\hline \multicolumn{2}{|c|}{ R Square } & \multicolumn{2}{|c|}{.021} & \multicolumn{2}{|c|}{.039} & \multicolumn{2}{|c|}{.058} \\
\hline \multicolumn{2}{|c|}{$\triangle \mathrm{R}$ Square } & & & \multicolumn{2}{|c|}{.018} & \multicolumn{2}{|c|}{.019} \\
\hline \multicolumn{2}{|c|}{$\mathrm{F}$} & \multicolumn{2}{|c|}{$15.596^{* * *}$} & \multicolumn{2}{|c|}{$15.236 * * *$} & \multicolumn{2}{|c|}{$15.162 * * *$} \\
\hline
\end{tabular}

${ }^{*} \mathrm{p}<.05,{ }^{* *} \mathrm{p}<.01, * * * \mathrm{p}<.001$

As for the moderating influence of resilience on environmental depression's effects on suicide intention, environmental depression positively affected suicide intention at Stage 1 . The explanatory power of the regression model increased by $1.8 \%$, with suicide intention positively affected by environmental depression and negatively affected by resilience at Stage 2 . The regression model's explanatory power increased by $1.9 \%$, with the interaction between environmental depression and resilience positively affecting suicide intention at Stage 3; that is, resilience moderated environmental depression's effects on suicide intention, as presented in [Table 10].

[Table 10] Analysis of the Moderating Influence of Resilience on Environmental Depression's Effects on Suicide Intention

\begin{tabular}{|c|c|c|c|c|c|c|c|}
\hline \multirow{2}{*}{$\begin{array}{c}\text { Dependent } \\
\text { variable }\end{array}$} & \multirow{2}{*}{$\begin{array}{c}\text { Independent } \\
\text { variable }\end{array}$} & \multicolumn{2}{|c|}{ Stage 1} & \multicolumn{2}{|c|}{ Stage 2} & \multicolumn{2}{|c|}{ Stage 3} \\
\hline & & $\boldsymbol{\beta}$ & $\mathbf{t}$ & $\beta$ & $\mathbf{t}$ & $\mathbf{p}$ & $\mathbf{t}$ \\
\hline \multirow{4}{*}{$\begin{array}{l}\text { Suicide } \\
\text { intention }\end{array}$} & (Constant) & & 2.799 & & 4.748 & & 5.636 \\
\hline & $\begin{array}{c}\text { Environmental } \\
\text { depression } \\
\text { (A) }\end{array}$ & .143 & $3.949 * * *$ & .092 & $2.383^{*}$ & -.305 & $-2.746 * *$ \\
\hline & $\begin{array}{l}\text { Resilience } \\
\text { (B) }\end{array}$ & & & -.147 & $-3.820 * * *$ & -.589 & $-4.814 * * *$ \\
\hline & $\mathrm{A} * \mathrm{~B}$ & & & & & .497 & $3.803 * * *$ \\
\hline \multicolumn{2}{|c|}{ R Square } & \multicolumn{2}{|c|}{.021} & \multicolumn{2}{|c|}{.039} & \multicolumn{2}{|c|}{.058} \\
\hline \multicolumn{2}{|c|}{$\triangle \mathrm{R}$ Square } & & & \multicolumn{2}{|c|}{.018} & \multicolumn{2}{|c|}{.019} \\
\hline \multicolumn{2}{|c|}{$\mathrm{F}$} & \multicolumn{2}{|c|}{$15.596 * * *$} & \multicolumn{2}{|c|}{$15.236 * * *$} & \multicolumn{2}{|c|}{$15.162 * * *$} \\
\hline
\end{tabular}

$* \mathrm{p}<.05, * * \mathrm{p}<.01, * * * \mathrm{p}<.001$

\subsubsection{Analysis of the Moderating Influence of Positive Psychological Capital on the Effects of Others'} Depression on Suicide Intention 
As for the moderating influence of self-efficacy on the effects of others' depression on suicide intention, others' depression positively affected suicide intention at Stage 1. The explanatory power of the regression model increased by $2.7 \%$, with suicide intention positively affected by others' depression and negatively affected by self-efficacy at Stage 2 . The regression model's explanatory power increased by $2.2 \%$, with the interaction between others' depression and self-efficacy negatively affecting suicide intention at Stage 3. Self-efficacy moderated the effects of others' depression on suicide intention, as presented in [Table 11].

[Table 11] Analysis of the Moderating Influence of Self-Efficacy on the Effects of Others' Depression on Suicide Intention

\begin{tabular}{|c|c|c|c|c|c|c|c|}
\hline \multirow{2}{*}{$\begin{array}{c}\text { Dependent } \\
\text { variable }\end{array}$} & \multirow{2}{*}{$\begin{array}{l}\text { Independent } \\
\text { variable }\end{array}$} & \multicolumn{2}{|c|}{ Stage 1} & \multicolumn{2}{|c|}{ Stage 2} & \multicolumn{2}{|c|}{ Stage 3} \\
\hline & & $\beta$ & $t$ & $\beta$ & $t$ & p & $\mathbf{t}$ \\
\hline \multirow{4}{*}{$\begin{array}{l}\text { Suicide } \\
\text { intention }\end{array}$} & (Constant) & & -.383 & & 4.683 & & -1.849 \\
\hline & $\begin{array}{c}\text { Others' } \\
\text { depression } \\
\text { (A) }\end{array}$ & .321 & $9.235^{* * *}$ & .331 & $9.786^{* * *}$ & .905 & $6.650 * * *$ \\
\hline & $\begin{array}{l}\text { Self-efficacy } \\
\text { (B) }\end{array}$ & & & -.221 & $-6.514 * * *$ & .149 & 1.632 \\
\hline & $A * B$ & & & & & -.712 & $-4.349 * * *$ \\
\hline \multicolumn{2}{|c|}{ R Square } & \multicolumn{2}{|c|}{.103} & \multicolumn{2}{|c|}{.151} & \multicolumn{2}{|c|}{.173} \\
\hline \multicolumn{2}{|c|}{$\triangle R$ Square } & & & \multicolumn{2}{|c|}{.048} & \multicolumn{2}{|c|}{.022} \\
\hline \multicolumn{2}{|c|}{$\mathrm{F}$} & \multicolumn{2}{|c|}{$85.286 * * *$} & \multicolumn{2}{|c|}{$66.236 * * *$} & \multicolumn{2}{|c|}{$51.528 * * *$} \\
\hline
\end{tabular}

As for the moderating influence of hope on the effects of others' depression on suicide intention, others' depression positively affected suicide intention at Stage 1 . The explanatory power of the regression model increased by $6.3 \%$, with suicide intention positively affected by others' depression and negatively affected by hope at Stage 2 . The regression model's explanatory power increased by $1.6 \%$, with the interaction between others' depression and hope negatively affecting suicide intention at Stage 3. Hope moderated the effects of others' depression on suicide intention, as presented in [Table 12].

[Table 12] Analysis of the Moderating Influence of Hope on the Effects of Others' Depression on Suicide Intention

\begin{tabular}{|c|c|c|c|c|c|c|c|}
\hline \multirow{2}{*}{$\begin{array}{l}\text { Dependent } \\
\text { variable }\end{array}$} & \multirow{2}{*}{$\begin{array}{l}\text { Independent } \\
\text { variable }\end{array}$} & \multicolumn{2}{|c|}{ Stage 1} & \multicolumn{2}{|c|}{ Stage 2} & \multicolumn{2}{|c|}{ Stage 3} \\
\hline & & $\beta$ & $\mathbf{t}$ & $\beta$ & $\mathbf{t}$ & p & $\mathbf{t}$ \\
\hline \multirow{4}{*}{$\begin{array}{l}\text { Suicide } \\
\text { intention }\end{array}$} & (Constant) & & -.383 & & 5.463 & & -.984 \\
\hline & $\begin{array}{c}\text { Others' } \\
\text { depression } \\
\text { (A) }\end{array}$ & .321 & $9.235^{* * *}$ & .317 & $9.438 * * *$ & .794 & $6.168 * * *$ \\
\hline & $\begin{array}{l}\text { Hope } \\
\text { (B) }\end{array}$ & & & -.250 & $-7.463 * * *$ & .081 & .878 \\
\hline & $A * B$ & & & & & -.591 & $-3.840 * * *$ \\
\hline \multicolumn{2}{|c|}{ R Square } & \multicolumn{2}{|c|}{.103} & \multicolumn{2}{|c|}{.166} & \multicolumn{2}{|c|}{.182} \\
\hline \multicolumn{2}{|c|}{$\triangle R$ Square } & & & \multicolumn{2}{|c|}{.063} & \multicolumn{2}{|c|}{.016} \\
\hline \multicolumn{2}{|c|}{$\mathrm{F}$} & \multicolumn{2}{|c|}{$85.286 * * *$} & \multicolumn{2}{|c|}{$73.630 * * *$} & \multicolumn{2}{|c|}{$54.912 * * *$} \\
\hline
\end{tabular}

$* * * \mathrm{p}<.001$ 
As for the moderating influence of optimism on the effects of others' depression on suicide intention, others' depression positively affected suicide intention at Stage 1. The explanatory power of the regression model increased by $6.4 \%$, with suicide intention positively affected by others' depression and negatively affected by optimism at Stage 2 . The regression model's explanatory power increased by $0.8 \%$, with the interaction between others' depression and optimism negatively affecting suicide intention at Stage 3; that is, optimism moderated the effects of others' depression on suicide intention, as presented in [Table 13].

[Table 13] Analysis of the Moderating Influence of Optimism on the Effects of Others' Depression on Suicide Intention

\begin{tabular}{|c|c|c|c|c|c|c|c|}
\hline \multirow{2}{*}{$\begin{array}{c}\text { Dependent } \\
\text { variable }\end{array}$} & \multirow{2}{*}{$\begin{array}{l}\text { Independent } \\
\text { variable }\end{array}$} & \multicolumn{2}{|c|}{ Stage 1} & \multicolumn{2}{|c|}{ Stage 2} & \multicolumn{2}{|c|}{ Stage 3} \\
\hline & & $\beta$ & $\mathbf{t}$ & $\boldsymbol{\beta}$ & $\mathbf{t}$ & $\mathbf{p}$ & $\mathbf{t}$ \\
\hline \multirow{4}{*}{$\begin{array}{c}\text { Suicide } \\
\text { intention }\end{array}$} & (Constant) & & -.383 & & 5.118 & & -.299 \\
\hline & $\begin{array}{l}\text { Others' } \\
\text { depression } \\
\text { (A) }\end{array}$ & .321 & $9.235^{* * *}$ & .348 & $10.342 * * *$ & .668 & $5.423 * * *$ \\
\hline & $\begin{array}{l}\text { Optimism } \\
\text { (B) }\end{array}$ & & & -.255 & $-7.575 * * *$ & -.007 & -.069 \\
\hline & $A * B$ & & & & & -.435 & $-2.695^{* *}$ \\
\hline \multicolumn{2}{|c|}{ R Square } & \multicolumn{2}{|c|}{.103} & \multicolumn{2}{|c|}{.167} & \multicolumn{2}{|c|}{.175} \\
\hline \multicolumn{2}{|c|}{$\triangle R$ Square } & & & \multicolumn{2}{|c|}{.064} & \multicolumn{2}{|c|}{.008} \\
\hline \multicolumn{2}{|c|}{$\mathrm{F}$} & \multicolumn{2}{|c|}{$85.286^{* * *}$} & \multicolumn{2}{|c|}{$74.565^{* * *}$} & \multicolumn{2}{|c|}{$52.550 * * *$} \\
\hline
\end{tabular}

$* * \mathrm{p}<.01, * * * \mathrm{p}<.001$

As for the moderating influence of resilience on the effects of others' depression on suicide intention, others' depression positively affected suicide intention at Stage 1. The explanatory power of the regression model increased by $3.7 \%$, with suicide intention positively affected by others' depression and negatively affected by resilience at Stage 2 . The regression model's explanatory power increased by $0.8 \%$, with the interaction between others' depression and resilience negatively affecting suicide intention at Stage 3; that is, resilience moderated the effects of others' depression on suicide intention, as presented in [Table 14].

[Table 14] Analysis of Moderating Influence of Resilience on the Effects of Others' Depression on Suicide Intention

\begin{tabular}{|c|c|c|c|c|c|c|c|}
\hline \multirow{2}{*}{$\begin{array}{c}\text { Dependent } \\
\text { variable }\end{array}$} & \multirow{2}{*}{$\begin{array}{l}\text { Independent } \\
\text { variable }\end{array}$} & \multicolumn{2}{|c|}{ Stage 1} & \multicolumn{2}{|c|}{ Stage 2} & \multicolumn{2}{|c|}{ Stage 3} \\
\hline & & $\beta$ & $\mathbf{t}$ & $\beta$ & $t$ & $\mathbf{p}$ & $\mathbf{t}$ \\
\hline \multirow{4}{*}{$\begin{array}{l}\text { Suicide } \\
\text { intention }\end{array}$} & (Constant) & & -.383 & & 4.096 & & -.438 \\
\hline & $\begin{array}{l}\text { Others' } \\
\text { depression } \\
\text { (A) }\end{array}$ & .321 & $9.235^{* * *}$ & .329 & $9.659 * * *$ & .669 & $4.847 * * *$ \\
\hline & $\begin{array}{l}\text { Resilience } \\
\text { (B) }\end{array}$ & & & -.193 & $-5.669 * * *$ & .023 & .249 \\
\hline & $A * B$ & & & & & -.420 & $-2.542 *$ \\
\hline \multicolumn{2}{|c|}{ R Square } & \multicolumn{2}{|c|}{.103} & \multicolumn{2}{|c|}{.140} & \multicolumn{2}{|c|}{.148} \\
\hline \multicolumn{2}{|c|}{$\triangle \mathrm{R}$ Square } & & & \multicolumn{2}{|c|}{.037} & \multicolumn{2}{|c|}{.008} \\
\hline \multicolumn{2}{|c|}{$\mathrm{F}$} & \multicolumn{2}{|c|}{$85.286^{* * *}$} & \multicolumn{2}{|c|}{$60.498 * * *$} & \multicolumn{2}{|c|}{$42.784 * * *$} \\
\hline
\end{tabular}

$* \mathrm{p}<.05, * * * \mathrm{p}<.001$ 


\section{Conclusion}

This study analyzed the moderating influence of positive psychological capital on geriatric depression's effects on suicide intention. The analysis obtained the following results:

First, geriatric depression affected suicide intention. This implies that the higher the level of geriatric depression, the stronger the intention to commit suicide. This result confirms that geriatric depression is the most important predictor of suicide and suggests the need to prevent depression to avoid elderly suicide.

Second, positive psychological capital partially affected suicide intention. Of the sub-variables of positive psychological capital, hope and optimism negatively affected suicide intention, and selfefficacy and resilience had no effect. Thus, the higher the level of hope and optimism, the weaker the intention to commit suicide, with self-efficacy or resilience having no effect on suicide intention. This implies that if seniors have hope and optimism about their lives and futures, they can circumvent suicide and lead a happy life with a healthy mind.

Third, depression partially affected positive psychological capital. The higher the level of selfdepression and environmental depression, the lower the level of positive psychological capital, with others' depression having no effect. The effects of depression on self-efficacy, self-depression, and environmental depression negatively affected self-efficacy, with others' depression having no effect. The effects of depression on hope, self-depression, environmental depression, and others' depression negatively affected hope. The effects of depression on optimism, self-depression, and environmental depression negatively affected optimism, with others' depression having no effect. The effects of depression on resilience, self-depression, and environmental depression negatively affected resilience, with others' depression having no effect.

Fourth, self-efficacy, hope, optimism, and resilience moderated the effects of self-depression on suicide intention. Meanwhile, self-efficacy, hope, optimism, and resilience moderated the environmental depression's effects on suicide intention, whereas self-efficacy, hope, optimism, and resilience moderated the effects of others' depression on suicide intention.

This study is significant because it determined the association between the effects of geriatric depression on suicide intention and positive psychological capital, and demonstrated the importance of positive psychological capital in preventing suicide in the elderly population. In particular, it is significant that it provided basic data required to develop interventional schemes and policies for elderly suicide prevention by demonstrating the moderating effects of positive psychological capital and intends to give implications for further research.

First, as geriatric depression is again identified as a variable affecting suicide intention, it is necessary to consider seniors at a high level of depression in a group at potentially high risk of suicide to prevent elderly suicide in elderly welfare practice effectively. It is, therefore, necessary to give them comprehensive, systematic service and build an arrangement that can help intervene rapidly in geriatric depression at an early stage and prevent it by building and connecting with personal and physical infrastructures, including elderly welfare centers, nursing homes, health centers, and mental health centers, which are frequented by the elderly.

Second, it is necessary to screen the high-risk elderly group of suicide. Minute assessment should be performed to determine the suicide intention, positive psychological capital, socio-demographic level, and depression confirmed in this study. Since the elderly may have many limitations related to communication with an interviewer and hand-writing in the screening process for suicide prevention service, screening based on the existing depression inventory can be limited. Therefore, mental health personnel needs to gather information through interviews with the elderly and people around them and cooperate with welfare centers, community service centers, and village heads to gather information to screen the high-risk group of suicide and prevent suicide effectively. Likewise, it is necessary to secure 
professionals that can build a system for managing the elderly in the high-risk group of suicide by generating life gate-keepers.

Third, the government needs to regard the mental health problems related to geriatric depression as social issues at the national level and form systems and policies for geriatric depression to prevent it from leading to suicide. To make this objective successful, efforts should improve nationwide awareness of geriatric depression and mental health via such channels as public service announcements, the Internet, or social media. Moreover, the government needs to help the elderly improve their mental health by supporting depression and suicide prevention programs in communitybased elderly welfare practice.

Fourth, it is necessary to allow seniors who are single or live alone to create further communication with their neighbors and increase personal contacts. The elderly living alone is likely to spend much time of the day without interpersonal relationships. The personal contacts with neighbors or friends are expected to relieve their sense of isolation and rebuild relational care. This would result in the restoration of their lost sense of belonging and effectively reduce suicidal ideation. In South Korea, local governments and private groups currently give a personal visit and home-based care, along with side dishes and yogurt, bath service, and elderly-elderly care for the elderly living alone and those with poor mobility. It is necessary to reflect this service in nationwide policies and make it mandatory to implement the service.

Fifth, since geriatric depression and suicide differ from the cases of other age groups, consideration should be given to their characteristics in the process and risk intervention programs. Since the elderly hesitate to visit any suicide prevention center for themselves, local governments and relevant centers need to visit and screen seniors exposed to the risk of suicide. The cases of the U.S. elderly mental health project (PROSPECT) demonstrated that based on the fact that $58 \%$ of the patients committing suicide visited a primary healthcare center one month before the suicide, first-line physicians screened depression patients and gave them medication and treatment with interpersonal relationships, reducing the suicide rate by a third; Japan ran community-based depression and suicide management programs to screen depression by asking questions to the elderly and giving education about suicide and depression and delivering guidebooks to detect the high-risk group of suicide and give them intensive care, reducing the suicide rate by a third. South Korea also needs to decrease the elderly suicide rate by making a drastic policy-based investment in elderly welfare and developing relevant programs.

Sixth, as positive psychological capital has been identified as a variable positively affecting geriatric depression and suicide intention, it is necessary to develop positive psychological capital programs that can help relieve and resolve suicide intention in the elderly population by building personal and physical infrastructures in health and welfare fields, as well as in public and private areas.

\section{References}

[1] D. B. Kim, B. S. You, A Study on the Neighborhood Environment, Social Relationship and Depression of the Elderly: In Case of Seoul Metropolitan Area, Journal of the Korean Gerontological Society, (2013), Vol.33, No.1, pp.105-123, UCI : G704-000573.2013.33.1.007

[2] http://www.mohw.go.kr/react/jb/sjb030301vw.jsp?PAR_MENU_ID=03\&MENU_ID=032901\&CONT_SEQ=361682, Dec 14 (2020)

[3] http://kostat.go.kr/portal/korea/kor_nw/1/1/index.board?bmode=read\&aSeq=385322, Sep 28 (2020)

[4]http://www.mohw.go.kr/react/jb/sjb030301vw.jsp?PAR_MENU_ID=03\&MENU_ID=032901\&CONT_SEQ=344953 \&page $=1$, May 30 (2018)

[5] M. R. Kim, The Effect of Psychosocial Factors on the Suicidal Ideation of Older Adults, Journal of Welfare for the 
Aged Institute, (2010), No.47, pp.113-136, I410-ECN-0102-2012-330-001022773

[6] I. J. Lee, Moderating Effects of Life Problems, Social Support on the Relationship between Depression and Suicidal Ideation of Older People, Health and Social Welfare Review, (2011), Vol.31, No.4, pp.34-62.

[7] S. A. Park, J. S. Hur, Moderating Effects of Religiosity on the Relationship between Depression and Suicidal Ideation of Older People, Journal of Welfare for the Aged, (2013), No.62, pp.79-108, I410-ECN-0102-2014-300-001621803

[8] M. E. P. Seligman, T. Rashid, A. C. Parks, Positive Psychotherapy, American Psychologist, (2006), No.61, pp.774778, https://doi.org/10.1037/0003-066X.61.8.774

[9] Y. E. Jung, M. J. Kang, G. Song, Y. S. Choi, J. M. Woo, J. H. Chae, Development of a Happiness Promoting Program based on Principles of Positive Psychology and Cognitive Behavioral Therapy-Focusing on Subjective Contentment, Cognitive Behavior Therapy in Korea, (2008), Vol.8, No.2, pp.51-62, UCI : G704-SER000008935.2008.8.2.001

[10] D. S. Lee, Y. D. Choi, A Study on Antecedents and Consequences of Positive Psychological Capital in Organizations, Korean Management Review, (2010), Vol.39, No.1, pp.1-28, UCI : G704-000126.2010.39.1.001

[11] K. K. Chon, M. K. Rhee, Preliminary Development of Korean Version of CES-D, Korean Journal of Clinical Psychology, (1992), Vol.11, No.1, pp.65-76.

[12] M. S. Shin, K. B. Park, K. J. Oh, Z. S. Kim, A Study of Suicidal Ideation among High School Students : The Structural Relation among Depression, Hopelessness, and Suicidal Ideation, Korean Journal of Clinical Psychology, (1990), Vol.9, No.1, pp.1-19.

[13] F. Luthans, B. J. Avolio, J. B. Avey, S. M. Norman, Positive Psychological Capital: Measurement and Relationship with Performance and Satisfaction, Personnl Psychology, (2007), Vol. 60, No.3, pp.541-572, https://doi.org/10.1111/j.1744-6570.2007.00083.x 